ISBN 978-93-84422-76-9

6th International Conference on Developments in Engineering and Technology

(ICDET-2017)

Bangkok (Thailand) Feb.6-7, 2017

\title{
On Microscopic Damping Evaluation of FRP Laminates
}

\author{
Dileep Kumar K ${ }^{1}$, Dr VV Subba Rao ${ }^{2}$

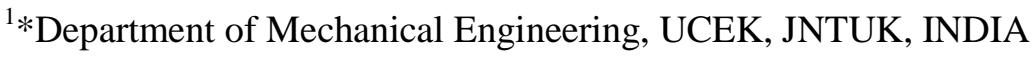 \\ ${ }^{2}$ Department of Mechanical Engineering, UCEK, JNTUK, INDIA \\ *Corresponding Author: e-mail: davidkumar999@jntucek.ac.in, Tel +91-9989707128
}

\begin{abstract}
The damping property of glass fiber reinforced polymer matrix composites with two different fiber strand diameters, orientations and layup sequence are investigated and an attempt is made to improve the damping of fiber reinforced polymer matrix composites by generating more number of interfaces. It is found that the damping will be improved at the negligible expense of stiffness, by generating more number of interfaces i.e reducing the fiber diameter from $27.2 \mu \mathrm{m}$ to $18.3 \mu \mathrm{m}$ without compromising the size of the composite specimen and the volume fraction of the fiber within the specimens. The loss factors are calculated from experimental results by using impulse technique. The same properties have also been evaluated theoretically by performing the two phase and three phase damping analysis using Ni and Adam's, Specific Damping Capacity (SDC) model, and the Interfacial Adhesion Model (IAM) respectively.
\end{abstract}

Keywords: Flexural vibration, composite laminates, specific damping capacity and loss factor.

\section{Introduction}

Damping of vibrations is an important aspect in the field of dynamic analysis of the engineering structures. The fiber reinforced composite materials are ideal structures wherever high strength-to-weight and stiffness-toweight ratios are demanded. Composite materials are often tailored to fulfill the desired stiffness and strength by changing lay-up and fiber orientations. The ability to tailor a material to its job is one among the most vital advantages of a composite material over a normal material. Therefore the research and development of composite materials becoming increasingly important for the weight sensitive application such as design of aerospace, automotive and marine structures also the change in its design aspects has grown tremendously within the past few decades. Damping in the structures is of many types, energy dissipation like material damping, aerodynamic damping and viscous damping etc. The current paper considers a discussion on the work related to material damping. The energy can be dissipated from the composite materials by different ways such as: the fiber-matrix interaction, visco-elastic behavior of the polymer composites, temperatures, damages, interfaces, and flexible bonding at fiber-matrix interface.

The energy can be dissipated from materials by different ways such as fibre orientation, visco elastic behavior of the PMC, temperatures, damages, interfaces, flexible bonding at fiber matrix interphase. In this work considered the interfaces for increasing the material damping. By creation of large number of interface regions the loss factor can be increased i.e.: fibre diameter is reduced without change in size of the laminate. For this work I considered fibers with diameters. If the fiber diameter decreases, the number of fiber-matrix interfaces increases, which leads to high energy dissipation when the specimen is excited. The Loss factor or specific damping capacity values have been found in the two different strands with the same fiber matrix proportions of the laminates. 
The analytical approach for the estimation of loss factors as a measure of microscopic damping is formulated under two different concepts the first is 2-phase microscopic damping and the second 3-phase microscopic damping. In which the loss factor of the lamina, a material system constitute of fiber and matrix considered as two phases and its damping nature determined by different models such as Adams and bacon, Adams and Maher, $\mathrm{Ni}$ and Adams and Lin- Tsai model. The loss factor of the 3-phase (an interface as an additional phase) is then computed by Wesley Gu,"interfacial adhesion model"to 2014 International Conference on Artificial Intelligence \& Manufacturing Engineering (ICAIME 2014). The Conference is a primary international forum for scientists and technicians working on topics relating to Artificial Intelligence \& Manufacturing Engineering. In the template, the Main Header1 will be of "Times New Roman" Font of 14 pt. each.

\section{Theoretical Calculation of the Damping}

\subsection{Three Phase Damping Model}

Contributions Damping can be increased by increasing the number of interfaces; it can be calculated by using three phase (fiber, matrix and interface) damping model. A three phase relation has been given in Eq.1. It contains the two-phase (fiber and matrix) loss factors and the interfacial loss factor of the composite materials, in which the two-phase loss factors are termed as system loss factor. Hence, the three phase relation has been used here to calculate the loss factor of the composite i.e the system and the interfacial loss factors are calculated separately.

$$
\eta_{c}=\eta_{f}+\eta_{m}+\eta_{i}=\eta_{s}+\eta_{m}
$$

Where $\eta \mathrm{c}=$ the loss factor of the composite,

$\eta i=$ the loss factor attributable to the interface,

$\eta \mathrm{s}=$ the loss factor attributable to the system (fiber +matrix),

$$
\begin{gathered}
E_{S_{L}}=E_{f_{(L)}} V_{f}+E_{m_{(L)}} V_{m} \\
\psi_{l 11}=\psi_{f 11} V_{f} \frac{E_{f 11}}{E_{l 11}}+\psi_{m n} V_{m} \frac{E_{m}}{E_{11}} \\
E_{22}=\frac{E_{m}}{1-\sqrt{k_{f}}\left(1-\frac{E_{m}}{E_{f 22}}\right)} \\
\psi_{l 22}=\psi_{f 22} \sqrt{k_{f}} \frac{E_{22}}{E_{f 22}}+\psi_{m n}\left(1-\sqrt{k_{f}}\right) \frac{E_{22}}{E_{m}} \\
G_{12}=\frac{G_{m}}{1-\sqrt{k_{f}}\left(1-\frac{G_{m}}{G_{f 12}}\right)} \\
\psi_{l 12}=\psi_{f 12} \sqrt{k_{f}} \frac{G_{12}}{G_{f 12}}+\psi_{m n}\left(1-\sqrt{k_{f}}\right) \frac{G_{12}}{G_{m}}
\end{gathered}
$$




$$
\gamma_{12}=v_{f} V_{f}+v_{m} V_{m}
$$

\subsection{Ni and Adams Theory}

The loss factors and the moduli of the system are evaluated only from the properties of the fiber and matrix. And also it can be effectively used to calculate the above properties of the system (the fiber and matrix) in the longitudinal, transverse and the longitudinal-transverse directions as given in the following section using rule of mixture. It is known that the term SDC $(\Psi)$ defines the ratio between energy dissipated to the total energy in cycle of vibration. The SDC model adopted here was developed by Ni and Adams assuming that the stressindependent damping coefficients are applicable at low, normal amplitudes, and a symmetric layup of the laminate. This final assumption leads to no midplane strains under classical laminate plate theory. In addition to neglecting oy and $\sigma x y, \mathrm{Ni}$ and Adams also argued that the transverse strain ey, in each lamina will be much smaller than the longitudinal and shear strains and could be neglected results eqs.

$$
\begin{aligned}
& \Psi_{1}=\frac{8 \Psi_{L}}{C_{11}^{*} N^{3}} \sum_{K=1}^{N / 2}\left(m^{2} C_{11}^{*}+m n C_{16}^{*}\right)\left[m^{2}\left(\overline{Q_{11}^{k}} C_{11}^{*}+\overline{Q_{12}^{k}} C_{12}^{*}+\overline{Q_{16}^{k}} C_{16}^{*}\right)\right] W_{K} \\
& \left.\Psi_{2}=\frac{8 \Psi_{T}}{C_{11}^{*} N^{3}} \sum_{K=1}^{N / 2}\left(m^{2} C_{11}^{*}-m n C_{16}^{*}\right)\left[n^{2}\left(\overline{Q_{11}^{k}} C_{11}^{*}+\overline{Q_{12}^{k}} C_{12}^{*}+\overline{Q_{16}^{k}} C_{16}^{*}\right)\right)\right] W_{K} \\
& \Psi_{12}=\frac{8 \Psi_{L T}}{C_{11}^{*} N^{3}} \sum_{K=1}^{N / 2}\left(2 m n C_{11}^{*}-\left(m^{2}-n^{2}\right) C_{16}^{*}\right)\left[m n\left(\overline{Q_{11}^{k}} C_{11}^{*}+\overline{Q_{12}^{k}} C_{12}^{*}+\overline{Q_{16}^{k}} C_{16}^{*}\right)\right] W_{K}
\end{aligned}
$$

Where $[\mathrm{C}]$ is the normalized flexural compliance of the laminate, [Qk] is the stiffness of the kth lamina, $\mathrm{p}$ is the total number of plies present in the laminate and $\mathrm{Wk}$ a weighting factor based on the position of the kth lamina within the laminate.

\subsection{Interfacial Damping}

The force required to overcome the frictional force in the longitudinal direction due to the radial pressure

$$
\frac{d F_{f}}{d x} d x=\mu p \cdot 2 \pi r_{f} d x
$$

Where $F_{f}$ is the axial force acting at the fiber, $r_{f}$ is the fiber radius, and $\mu$ is the friction coefficient between fiber and matrix. Similarly, if the interfacial deboning is caused by the force $F_{f}$ during the stretching of the composite, then $\mu p$ in Equation can be replaced by the interfacial shear strength of the composite, $\tau$, which is that necessary to pull the fiber from the matrix.

$$
\frac{d F_{f}}{d x}=2 \pi r_{f} \tau
$$

By integrating the above equation (11) we will get

$$
F_{f}=2 \pi r_{f} \tau x
$$

From the above equation the deboning length can be written as

$$
x=\frac{F_{f}}{2 \pi r_{f} \tau}
$$

For a cantilever beam, if there is an interfacial deboning, it likely occurs at the free end of the beam because shear strain (and shear stress) is high at that location. In this case, the force balance is

The maximum debonding region is caused by the maximum load on fiber $F_{f}^{\max }$. When an alternating. Load is applied to the composite, such as in the case of vibration

$$
I_{c}=b h^{3} / 12
$$

When the beam is deflected by an end-applied load $P$, the shear component of the load can be obtained 


$$
F=F_{f} \approx P \theta_{0}=\frac{P^{2} L^{2}}{2 E_{c} I_{c}}
$$

Where $I_{c}$ is the moment of inertia of the composite beam. Therefore, the maximum load carried by fiber $F_{f}^{\max }$ corresponding to the maximum bending load, $P_{\max }$, can be expressed quantitatively as

$$
F_{f}^{\max }=\frac{P_{\max }^{2} L^{2}}{2 E_{c} I_{c}}
$$

The energy dissipation in a cycle $i$ s small compared to the maximum stored elastic energy $W$ of the system, which is true for most vibration damping situations, the interfacial damping factor, $\tan \delta_{i n}$, may be estimated by the following equation

$$
\tan \delta_{i n}=\frac{\Delta W}{2 \pi W}
$$

Assuming the stress in the fiber gradually approaches the maximum value (or $P$ gradually reaches to $\mathrm{P}_{\max }$ ) during a vibration cycle, interfacial deboning and slippage between fiber and matrix will dissipate energy because the integration is performed over a complete cycle about the origin, the energy dissipated per cycle is given by

$$
\begin{gathered}
\Delta W=2 F_{f}^{\max } x_{0} \\
\Delta W=\frac{P_{\max }^{4} L^{4}}{4 \pi r_{f} \tau\left(E_{c} I_{c}\right)^{2}}
\end{gathered}
$$

The potential energy $W$ for a sample subjected to strain can be calculated as follows

$$
W=\int_{0}^{\varepsilon_{\max }}\left(\sigma_{c} A_{c}\right) d \varepsilon L=\frac{1}{2} A_{c} L E_{c} \varepsilon_{\max }^{2}
$$

Where $L$ is the sample length, and $I_{c}, A_{c}$, and $E_{c}$ are the stress, cross-sectional area, and Young's modulus of the composite, respectively. For a cantilever beam

$$
W=\frac{1}{2} A_{c} L E_{c}\left(\frac{P_{\max }^{2} L^{4}}{15\left(E_{c} I_{c}\right)^{2}}\right)^{2}
$$

By substituting Equations (4.1.69) and (4.1.71) into Equation (4.4.67), and by knowing that, $\boldsymbol{I}_{c}=\boldsymbol{b} \boldsymbol{h}^{3} / \mathbf{1 2} \mathrm{we}$ obtain

$$
\tan \delta_{i n}=\frac{50 E_{c} b h^{5}}{64 \pi^{2} \pi d f L^{5}}
$$

Where $b$ is the sample width and $h$ is sample thickness.

$$
\left(E_{s_{L}}, E_{s_{T}}, E_{s_{L T}}, \eta_{s_{L}}, \eta_{s_{T}}, \eta_{s_{L T}}, v_{s_{L T}}\right)
$$

Where $\eta_{i}$ is the interfacial loss factor

$\mathrm{E}_{\mathrm{c}}$ is the longitudinal modulus of the system (fiber and matrix),

$\mathrm{b}, \mathrm{h}$ and $\mathrm{l}$ are the width, depth and length of the single fiber specimen,

$\mathrm{T}$, is the interface shear strength,

$\mathrm{d}_{\mathrm{f}}$ is the fiber diameter.

As the current study mainly concentrates on the effect of the variation of the two different diameters of the fiber, the diameters have been accurately measured, using the SEM images captured from these two categories of specimens in which the sizes are measured around $18.3 \mu \mathrm{m}$ and $27.2 \mu \mathrm{m}$ for the small and large fiber diameters.

For a small and large diameters of fiber the interfacial loss factors can be written as per a small fiber diameter 


$$
\eta_{i_{l(\theta=0 t o 90)}}==\frac{50 E_{c_{l(\theta-0 t o 90)}} b h^{5}}{64 \pi^{2} \tau d_{f_{l}} L^{5}}
$$

For a large fiber diameter

$$
\eta_{i_{l(\theta=0 t o 90)}}==\frac{50 E_{c_{l(\theta \theta=0 t o 90)}} b h^{5}}{64 \pi^{2} \tau d_{f_{l}} L^{5}}
$$

Where, $\eta_{i_{s}}$ and $\eta_{i_{\iota_{1}}}$ are the interfacial loss factors of the small and large fibers respectively

$\boldsymbol{E}_{c_{s}}$ and $\boldsymbol{E}_{c_{l}}$ are the longitudinal moduli of the system (fiber and matrix) with the small and large fibers respectively,

$d_{f_{s}}$ And $d_{f_{i}}$ are the diameters of the small and large fibers respectively.

Since the interface damping equations provided in Eqs74. Are applicable only for the $0^{\circ}$ orientation of the fiber in the longitudinal direction, the same has been extended to the different orientations $\left(30^{\circ}, 45^{\circ}, 60^{\circ}\right.$ and $\left.90^{\circ}\right)$ of the fiber as well.As the longitudinal modulus values are decreased with the orientations of the fiber, those modulus values are calculated based on the transformation relations provided in the Eq of The interfacial loss factors with respect to the different orientations of the small and large fibers have been calculated using the following equations.

$$
\begin{gathered}
\eta_{i_{(\theta \theta=0 t o 90)}}==\frac{50 E_{c_{s(\theta \theta-t o \rho 90)}} b h^{5}}{64 \pi^{2} \tau d_{f_{s}} L^{5}} \\
\eta_{i_{l(\theta=t o 90)}}==\frac{50 E_{c_{l(\theta=0 t o 90)}} b h^{5}}{64 \pi^{2} \tau d_{f_{l}} L^{5}}
\end{gathered}
$$

Where, $\eta_{i_{s(\ell=0 t o 90)}}, \eta_{i_{l}(\ell=0 t o 90)}$ are the interfacial loss factors of the small and large fibers in longitudinal direction and the different orientations of fibers.

After the calculations of the system and interfacial loss factors $\left(\eta_{s}\right.$ and $\left.\eta_{1}\right)$ of the small and large fiber diameters, the theoretical loss factors $\left(\eta_{c}\right)$ are calculated from Eq (25) and (26).

TABLE I: The Material Properties of the Fiber and Matrix for the Small and Large Fibre Diameters

\begin{tabular}{|c|c|c|c|c|c|c|c|}
\hline Materials & $\begin{array}{c}\text { Longitudinal } \\
\text { Modulus (GPa) }\end{array}$ & $\begin{array}{c}\text { Transverse } \\
\text { Modulus } \\
\mathbf{G P a}\end{array}$ & $\begin{array}{c}\text { Shear } \\
\text { Modulus } \\
\mathbf{( G P a )}\end{array}$ & $\begin{array}{c}\text { Longitudinal } \\
\text { Loss factor \% }\end{array}$ & $\begin{array}{c}\text { Transverse } \\
\text { Loss factor \% }\end{array}$ & $\begin{array}{c}\text { Longitudinal } \\
\text { Transverse Loss } \\
\text { factor \% }\end{array}$ & $\begin{array}{c}\text { Poisson's } \\
\text { Ratio }\end{array}$ \\
\hline $\begin{array}{c}\text { Small dia. D= } \\
17 \mu \mathrm{m}\end{array}$ & 60.54 & 60.54 & 25.44 & 0.1749 & 0.1749 & 0.09533 & 0.2 \\
\hline $\begin{array}{c}\text { Large dia. } \\
\mathrm{D}=27 \mu \mathrm{m}\end{array}$ & 61.07 & 61.07 & 25.44 & 0.1749 & 0.1749 & 0.09533 & 0.2 \\
\hline Matrix Fiber & 2.737 & 2.737 & 1.015 & 1.3989 & 1.3989 & 1.5788 & 0.374 \\
\hline
\end{tabular}

\section{Results and Discussions}

The damping value can only be achieved at the expense of stiffness. Since a high damping value is desired to be obtained at a smaller expense of the stiffness value, it is done by generating more number of interfaces (i.e. more number of the same can be created by reducing the strand diameter/diameter of the fiber, without changing the volume fraction of the fiber and the matrix) under different stacking sequences of the fiber, which leads to a larger energy dissipation. It is evident from Figs below that the different strand diameters of the fiber under different stacking sequences greatly influence its dynamic behavior .It is also noted that the loss factor and the natural frequency/stiffness are high in the unidirectional ply of the fiber at $90^{\circ}$ and $0^{\circ}$ orientations respectively. 


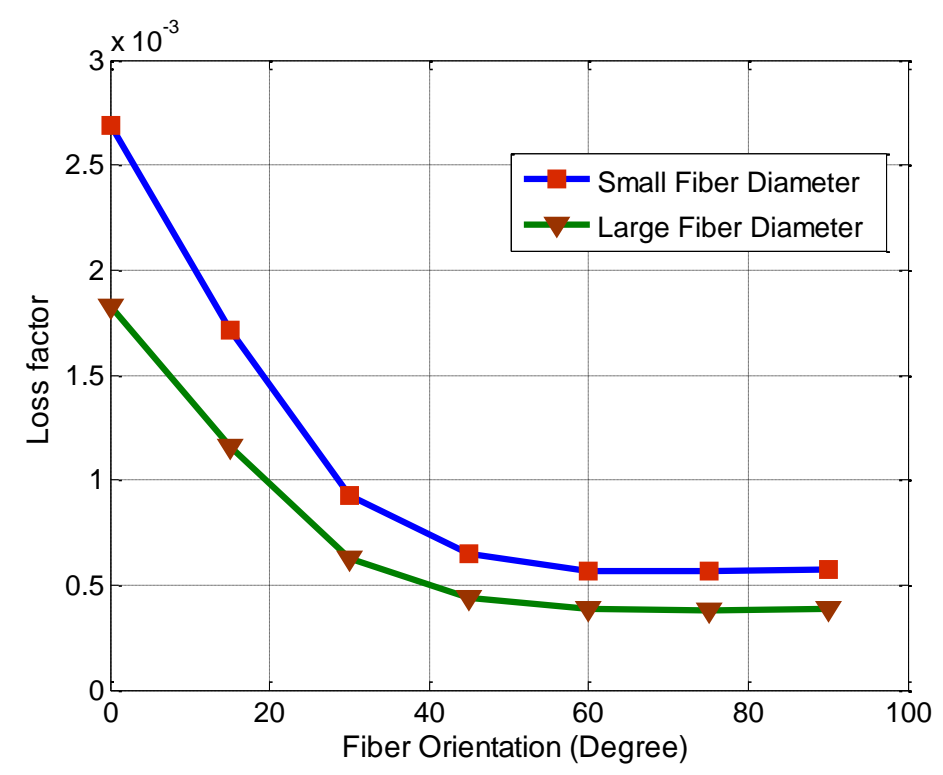

Fig1: Three phase loss factors for small fibrediameters

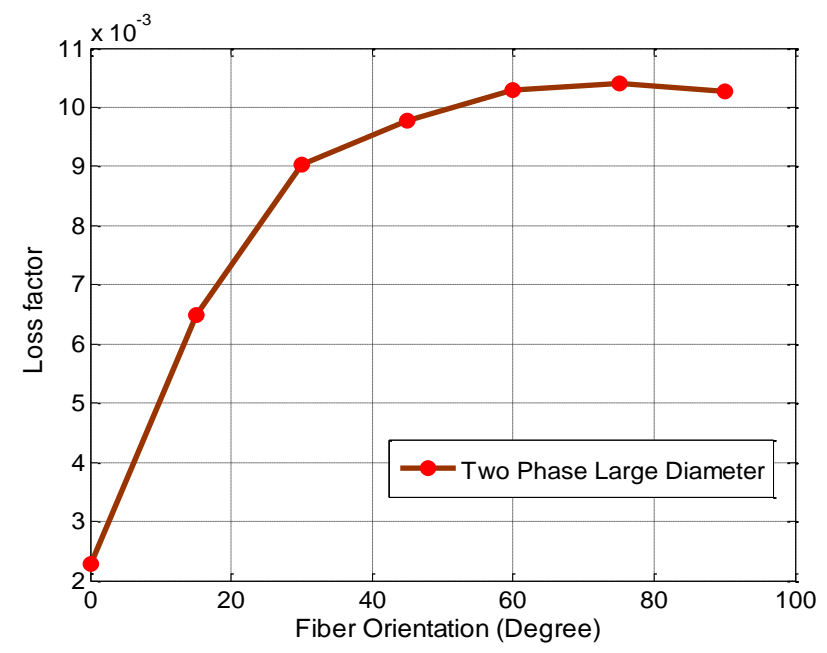

Fig. 2 : Interfacial loss factors for small and large fibre diameters

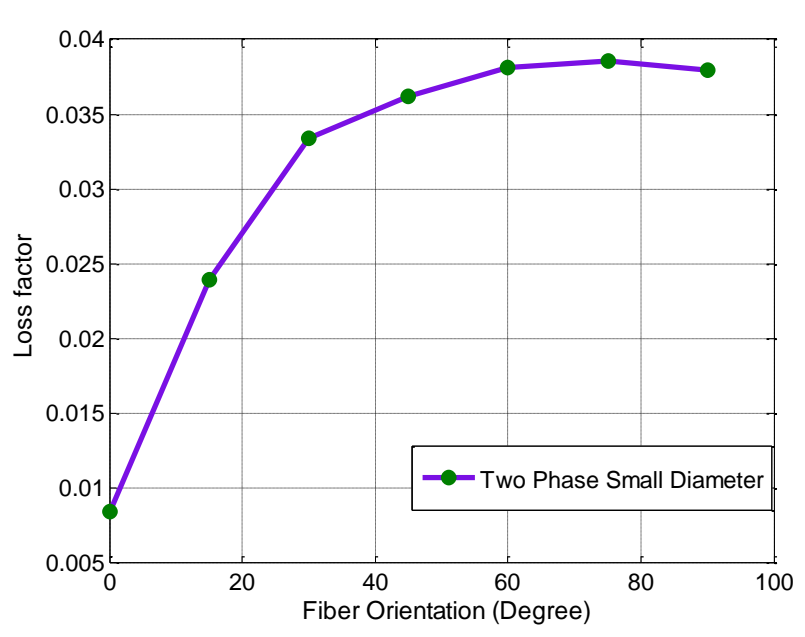

Fig. 3 : Two phase loss factors for small fibre diameters

\section{Concluions}

Investigating the dynamic behavior of GFRP composites, aiming to improve the damping without compromising much on the magnitude of stiffness/frequency. Two different diameters of the glass fiber with $0^{0}$, $30^{\circ}, 45^{\circ}, 60^{\circ}$ and $90^{\circ}$ orientations in the polymer matrix were fabricated. After carrying out detailed studies of the frequency and the damping experimentally and theoretically, it is concluded that the laminate with the small diameter and $45^{\circ}$ orientation of the fiber exhibits better results. Even this small reduction in diameter has caused approximately an average of $18 \%$ increase from the overall damping (3phasedamping) model. In case the fiber diameter differences are high, the percentage increase in the damping can also be raised. After evaluating the 3phase damping i.e. including the interface damping, the variation between the theoretical and the experimental damping values has been significantly reduced. 


\section{References}

[1] Ni RG, Adams RD. The damping and dynamic moduli of symmetric laminated composite beams-theoretical and experimental results. J Compos Mater1984;18(2):104-21.

https://doi.org/10.1177/002199838401800202

[2] Adams RD, Maheri MR. Dynamic flexural properties of anisotropic fibrous composite beams. Compos Sci Technol 1994;50(4):497-514.

https://doi.org/10.1016/0266-3538(94)90058-2

[3] Adams RD, Bacon DGC. Effect of fibre orientation and laminate geometry on the dynamic properties of CFRP.J Compos Mater 1973; 7:402-28.

https://doi.org/10.1177/002199837300700401

[4] Saravanos DA, Chamis CC. Mechanics of damping for fiber composite laminates including hygrothermal effects. AIAA $J$ 1990;28(10):1813-9. https://doi.org/10.2514/3.10478

[5] Berthelot J-M, Sefrani Y. Damping analysis of unidirectional glass and Kevlar fibre composites. Compos SciTechnol 2004;64(9):1261-78. https://doi.org/10.1016/j.compscitech.2003.10.003

[6] Berthelot J-M. Damping analysis of laminated beams and plates using the Ritz method. Compos Struct 2006;74:186201. https://doi.org/10.1016/j.compstruct.2005.04.031

Ohta Y, Narita Y, Nagasaki K. On the damping analysis of FRP laminated composite plates. Compos Struct 2002;57:169-75.

https://doi.org/10.1016/S0263-8223(02)00080-6

[7] Yim JH, Gillespie Jr JW. Damping characteristics of 0 and 90 AS4/3501-6 unidirectional laminates including the transverse shear effect. Compos Struct.2000;50:217-25.

https://doi.org/10.1016/S0263-8223(00)00087-8

[8] Wei YT, Gui LJ, Yang TQ. Prediction of the 3-D effective damping matrix of viscoelastic fiber composites. Compos Struct 2001;54:49-55.

https://doi.org/10.1016/S0263-8223(01)00069-1

[9] Billups EK, Cavalli MN. 2D damping predictions of fiber composite plates:layup effects. Compos SciTechnol 2007;68:727-33.

https://doi.org/10.1016/j.compscitech.2007.09.007

[10] Chandra R, Singh SP, Kupta K. Damping studies in fiber-reinforced composites- a review. Compos Struct 1999;46:4151.

https://doi.org/10.1016/S0263-8223(99)00041-0

[11] Ungar EE, Kerwin EM. Loss factors of viscoelastic systems in terms of strain energy AcoustSoc Am 1962;34(2):954 